\title{
"SILAB for Africa": An Innovative Information System Supporting the Veterinary African Laboratories
}

\author{
Patrizia Colangeli, MS, ${ }^{1}$ Ercole Del Negro, $M S^{1}$ \\ Umberto Molini, VD ${ }^{2}$ Sara Malizia, $M S^{1}{ }^{1}$ \\ and Massimo Scacchia, $V D^{3}$ \\ ${ }^{1}$ Information System management Unit -ICT Department, \\ Veterinary Public Health Institute of Abruzzo and Molise \\ Regions (IZSAM), Teramo, Italy. \\ ${ }^{2}$ Central Veterinary Laboratory, Windhoek, Namibia. \\ ${ }^{3}$ Animal health Department, Veterinary Public health Institute \\ of Abruzzo and Molise Regions (IZSAM), Teramo, Italy.
}

\section{Abstract}

To support African veterinary laboratory services, the Istituto Zooprofilattico Sperimentale dell'Abruzzo e del Molise puts in place an operational system called "SILAB for Africa" (SILABFA); this is a web application used by a laboratory information management system to support laboratory diagnostic activities and to meet the needs of various African countries. SILABFA was designed to collect and manage all necessary information on samples, tests, and test results. The system involves the entry of sample data on arrival, the tracking of samples through the various sections of the laboratory, and the collection of test results. It automates the generation of test reports and monitors outbreaks through data interrogation functions and eliminates multiple registrations of the same data on paper records. SILABFA is currently installed in Namibia, Botswana, Zambia, Zimbabwe, Tanzania, Uganda, Kenya, Ethiopia, and Cameroon, and installation in Senegal and Ivory Coast is planned for the next few months. After some years of SILABFA usage, it was natural to want to utilize more and more data collected in a homogeneous and consistent way for epidemiological purposes and to cover informative debts toward ministries and other organizations. To improve the availability of good, detailed, and reliable data, as the epidemiological information, SILABFA has been linked to the local animal identification, registration, and traceability system and other relevant national information systems.

Keywords: information management, e-Health, business administration, economics, technology

\section{Introduction}

$\mathrm{n}$ the past decades, as many as $60 \%$ of all communicable diseases and 75\% of emerging infectious diseases of people originated with animals. Zoonoses can be caused by bacteria, fungi, mycobacteria, parasites, viruses, and prions. ${ }^{1}$

Using a One-Health Approach, the World Organization for Animal Health (OIE), the World Health Organization (WHO), and the Food and Agriculture Organization of the United Nations (FA0) are coordinating global activities to address health risk at human-animal-ecosystem interface. In several programs supported by these organizations, the significance of the Regional Laboratory Network in supporting disease detection, surveillance, and response to disease outbreaks is recognized.

In particular, laboratory information management system (LIMS) is recognized as a powerful tool to improve laboratory data management within the laboratories and to report data externally. LIMS is often expensive and a long-time investment; for these reasons, it has not been deployed in a significant proportion in veterinary laboratories in developing countries, limiting its capacity to contribute adequately to the surveillance of, and response to, public health events of international concern such as epidemics.

In this context, the Information System management Unit (ICT) of the Veterinary Public Health Institute of Abruzzo and Molise Regions (IZSAM) in 2009 developed a LIMS called "SILAB for Africa" (SILABA) with the purpose of supplying an information system for laboratory management to the Windhoek's Central Veterinary Laboratory (CVL)-Namibia. ${ }^{2}$

The advantages obtained by the Namibian laboratory by the constant utilization of SILABFA (among them, the ISO 17025 accreditation obtained in 2013) have driven an interest from other neighboring countries to request for the same application. In 2012, SILABFA was installed in Botswana, Zimbabwe, and Zambia. In 2013, FA0 cofinanced the extension of SILABFA in Botswana and its interconnection with the national animal identification and traceability system (BAITS). FAO also supports the installation of the system in the Tanzanian Veterinary Laboratory Agency (TVLA) in Dar es Salaam, Arusha, and Iringa. Afterward, two new agreements between FAO and IZSAM were signed for the installation of 


\section{COLANGELI ET AL.}

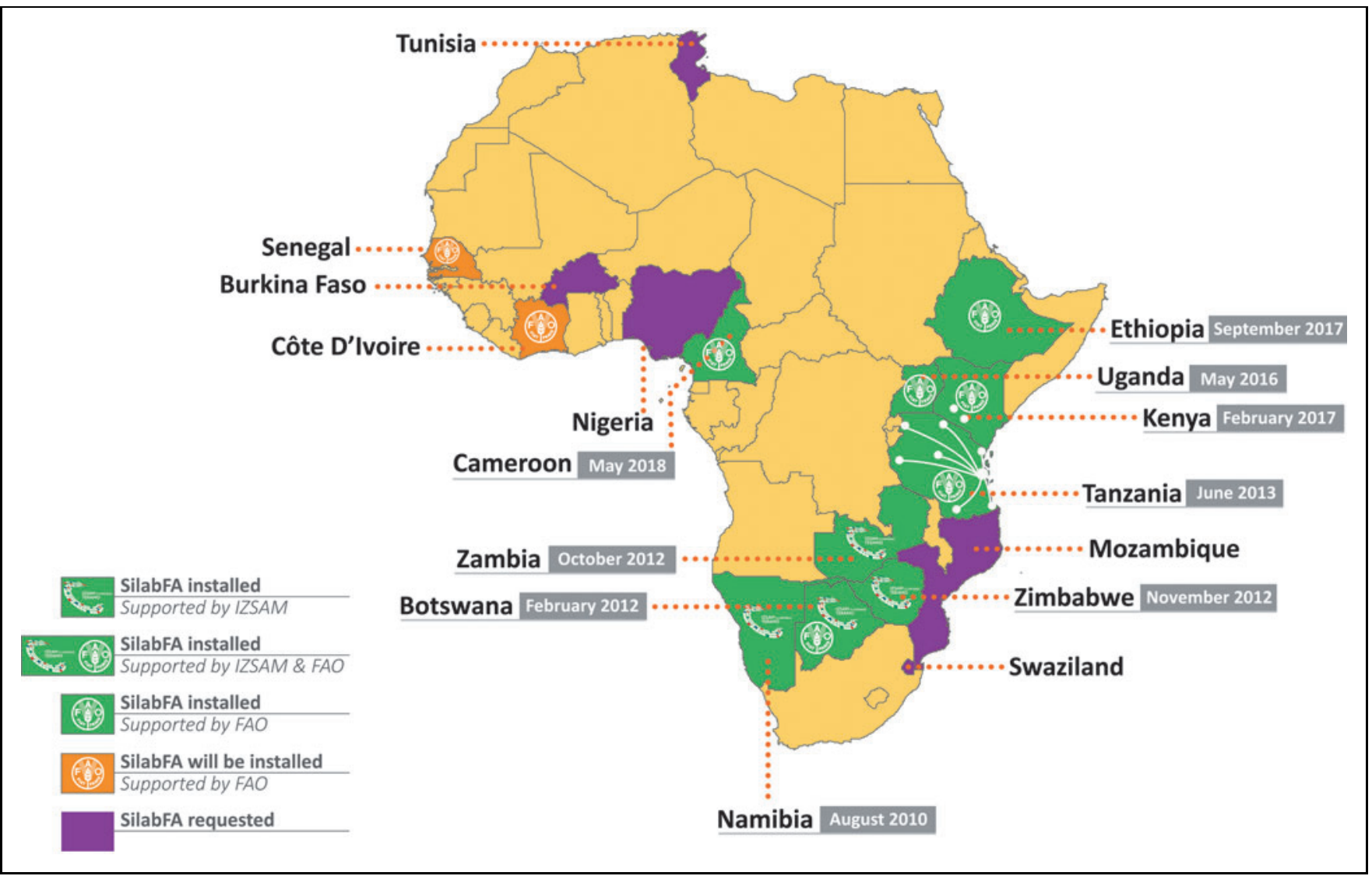

Fig. 1. SILABFA installations in Africa in May 2018. SILABFA, "SILAB for Africa."

SILABFA in Uganda, Kenya, Ethiopia, Cameroon, Senegal, and Ivory Coast. Currently, nine African countries are using SILABFA on a regular basis (Fig. 1). By 2019, it is projected that 11 African countries will be using the system.

IZSAM does not play the role of supplying software, but has been able to enter into lasting relationships. The collaborative approach of IZSAM personnel has allowed to create reciprocal trust and a more profound functioning of the African partners.

Therefore, after few years of SILABFA's usage, some involved countries shared a belief that it is an essential element in the entire veterinary information system, which allows utilizing more and more data collected for epidemiological purposes, and to provide information toward ministries and other organizations, avoiding redundancies and inefficiencies.

In addition, the efficiency of any laboratory and in particular accreditation (according to ISO/IEC 17025 standards) are a prerequisite for countries to access international trade.

Finally, interlinkage and interoperability between SILABFA and the animal identification, registration, and traceability system (AIRTS) (e.g., BAITS in Botswana, Namibian Livestock Information Traceability System (NAMLITS) in Namibia, TANLITS in Tanzania) can improve greatly the data quality of the entire cycle of livestock production, from the field to the laboratory and the export process.

Such interoperability allows to insert data just once, in one system, and to make data automatically available to other systems enabling rapid and fluid data transfer, in particular, from or to the laboratories and from or to veterinary services. This increases the effectiveness and efficiency of services involved in this whole chain, which enhances the exchange of information and promotion of a better communication among operators.

The first connection between SILABFA and AIRT databases was made in Botswana in 2014. The connection between SILABFA and NAMLITS has been put in place in Namibia since August 2015 and the connection with TANLITS was already examined using web services solution but has yet to be realized.

\section{Materials and Methods}

SILABFA is a web-based application, developed utilizing exclusive open source and freeware software. The application is developed with Java technologies and Apache Tomcat as web server. The relational database (RDBMS) is Oracle 11g Express Edition. The features of this freeware RDBMS 


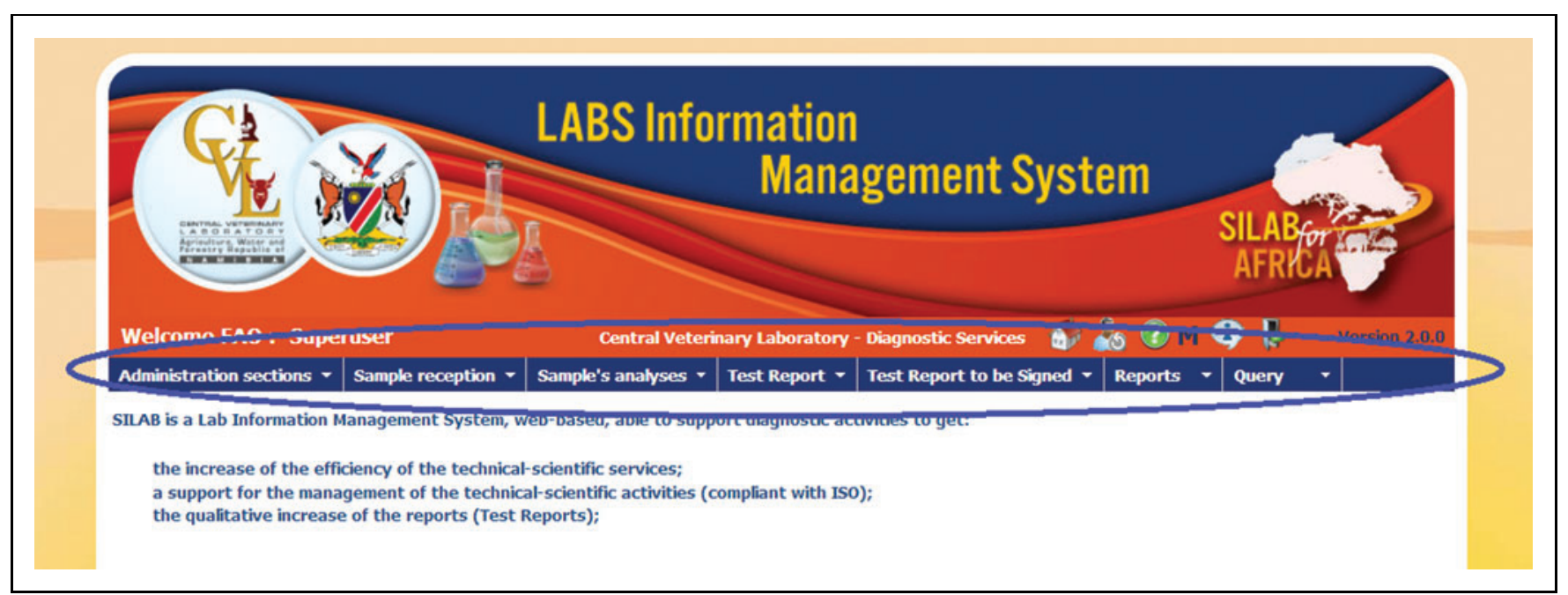

Fig. 2. SILABFA home page and the main functionalities.

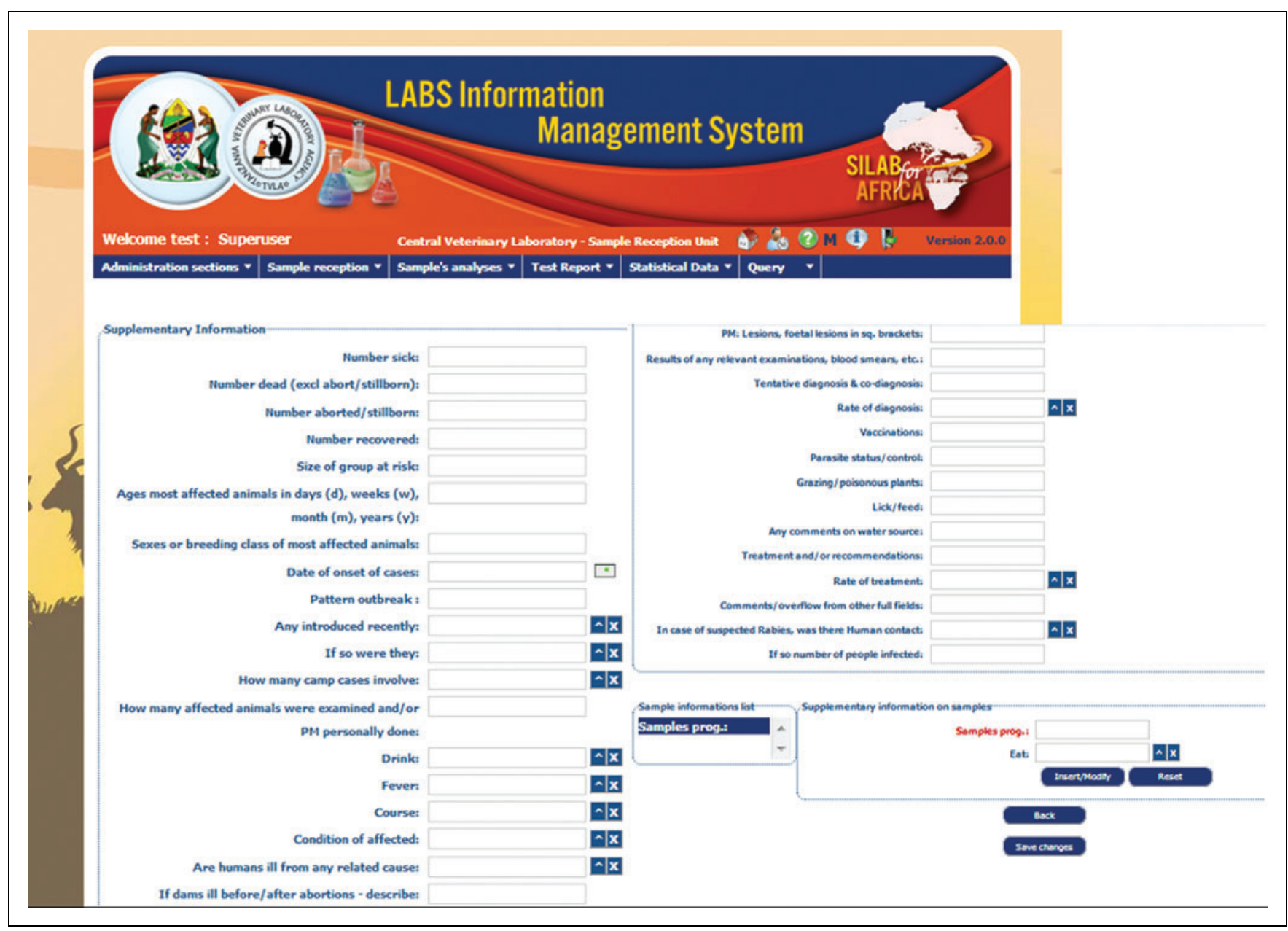

Fig. 3. Form to enter additional information according to Namibian rabies plan. 


\section{COLANGELI ET AL.}

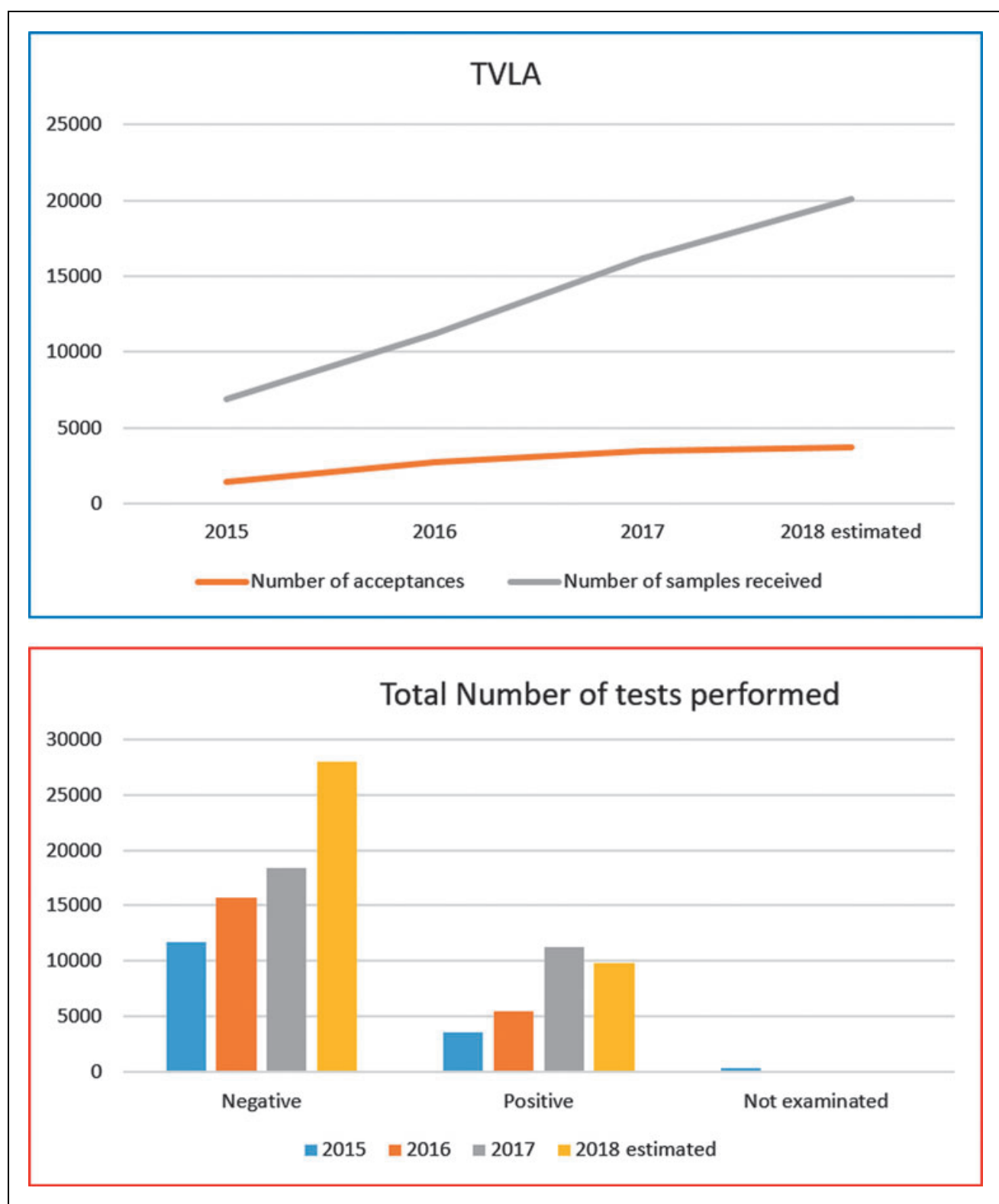

Fig. 4. Tanzanian laboratory-TVLA trend in terms of acceptances, samples received, and tests performed. TLVA, Tanzanian Veterinary Laboratory Agency.

(11 gigabyte of storage, 1 gigabyte of utilized memory, and 1 CPU) are sufficient to guarantee all functionalities. The report system tool is Jasper Report, capable to generate any file format. Thanks to these choices, SILABFA is distributed for free.

System's installation requires a server connected to a local area network (LAN), reachable by any computers in the laboratory. Any costs for server and LAN are up to the beneficiary country as well as the availability of local IT person. For SILAB use, internet connection is not necessary but it became mandatory for distributed access (like in Zambia and Tanzania), for interconnection among external databases (Botswana and Namibia) and to allow remote support (possible by smartphone). Costs for internet connections have to be covered by beneficiary countries.

Data integrity is guaranteed by using development standards as well as by the security of the network. All data are saved and stored according to a well-defined backup procedure and disaster recovery procedures, which are available to the local IT unit.

IZSAM personnel guarantees SILABFA installation on a local server, and in collaboration with local personnel, it provides the first database loading (information of users, roles of the users, tests, methods, SOPs, etc.). The training phase (free for beneficiary countries) is organized locally using on-the-job-training method, and it lasts for 3 weeks. During this pilot phase, SILABFA is utilized at the office reception and in two to three departments, in parallel with the hard copy system. At the end of this training, local users are able to independently extend SILABFA to all other departments in the laboratory.

In parallel with the local installation, IZSAM, using own servers in Italy, provides for each country self-training session and beta version of new functions before their installation.

IZSAM assures a daily support and offers new upgrades of the system (without charge for countries). Annual audit visit to each country (funded by FAO or IZSAM) is guaranteed to verify quality standards and to collect new needs. To assure privacy and property of data, a confidentiality agreement between IZSAM and the laboratory is signed.

\section{Results}

SILABFA allows the insertion of all data characterizing the sampling activity, including additional information expected in surveillance and monitoring plans for each country. It also 


\section{SILABFA: A LIMS FOR VETERINARY LABORATORIES}

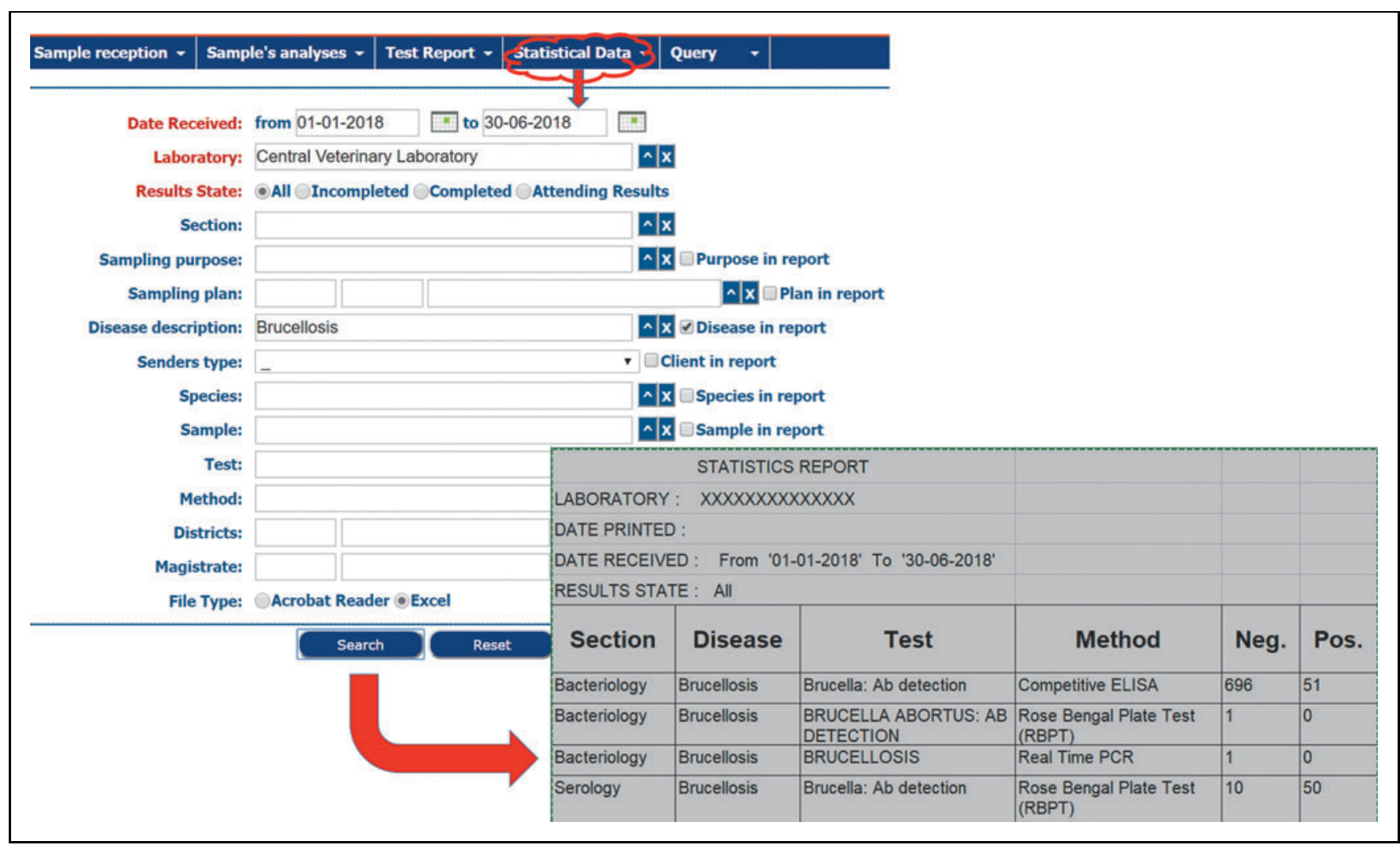

Fig. 5. Statistical form and results.

allows the traceability of any diagnostic path in different sections, input of test results, generation of test reports with automatic signature, and sending results through e-mail to customers. Moreover, SILABFA offers data query and rapid alert functions that are useful for outbreak alert, and helps avoid multiple manual registrations (Fig. 2).

Since 2014, SILABFA in Botswana has been interfaced successfully with BAITS database. BAITS ensures that each cattle is individually identified and traced throughout its life. Over 200,000 livestock (bovine) keeper information is automatically caught by the BAITS database, as well as owners, feedlots, holdings, abattoirs, slaughterhouses, and ranches. To simplify the interconnection, the SILABFA database codes are realigned to the BAITS database codes: the regions, districts, external areas, the type of sample, and the animal species have the same codes in both systems. The connection is safe and fast because the BAITS is installed on a server located in the Botswana Ministry of Agriculture headquarters, in the same domain of SILABFA server (gov.bw), the systems are connected using a database link (DBLINK).

The NAMLITS is a mandatory tool used at farm level to monitor and manage herd performance. It is used to docu- ment animal health status, reproductive history, and movement of animals in the country, and meet market entry and traceability requirements of livestock and products of animal origin throughout the entire supply chain. The interoperability between SILABFA and NAMLITS was funded by Namibia's CVL and has been on-line since September 2015. All information stored in the NAMLITS database regarding the origin of samples (keeper, farm name, location, animal_ID, animal category, etc.) can be found in the SILABFA database. This interoperability could work in two ways as SILABFA can send to NAMILITS the results related to a herd sampling activity or specific bovine health status.

Quality standards that SILABFA guarantees (in compliance with ISO/IEC 17025, among others) help to achieve important results in each laboratory. These standards facilitated the accreditation of the laboratories in Namibia and Zambia and its retention and improvement in Botswana, Zimbabwe, and Ethiopia laboratories.

Collected data are used not just to produce test reports but also for epidemiological purposes, for example, the management of national plans of disease control like the control plan of rabies that the Namibian government instituted in 2015. 


\section{COLANGELI ET AL.}

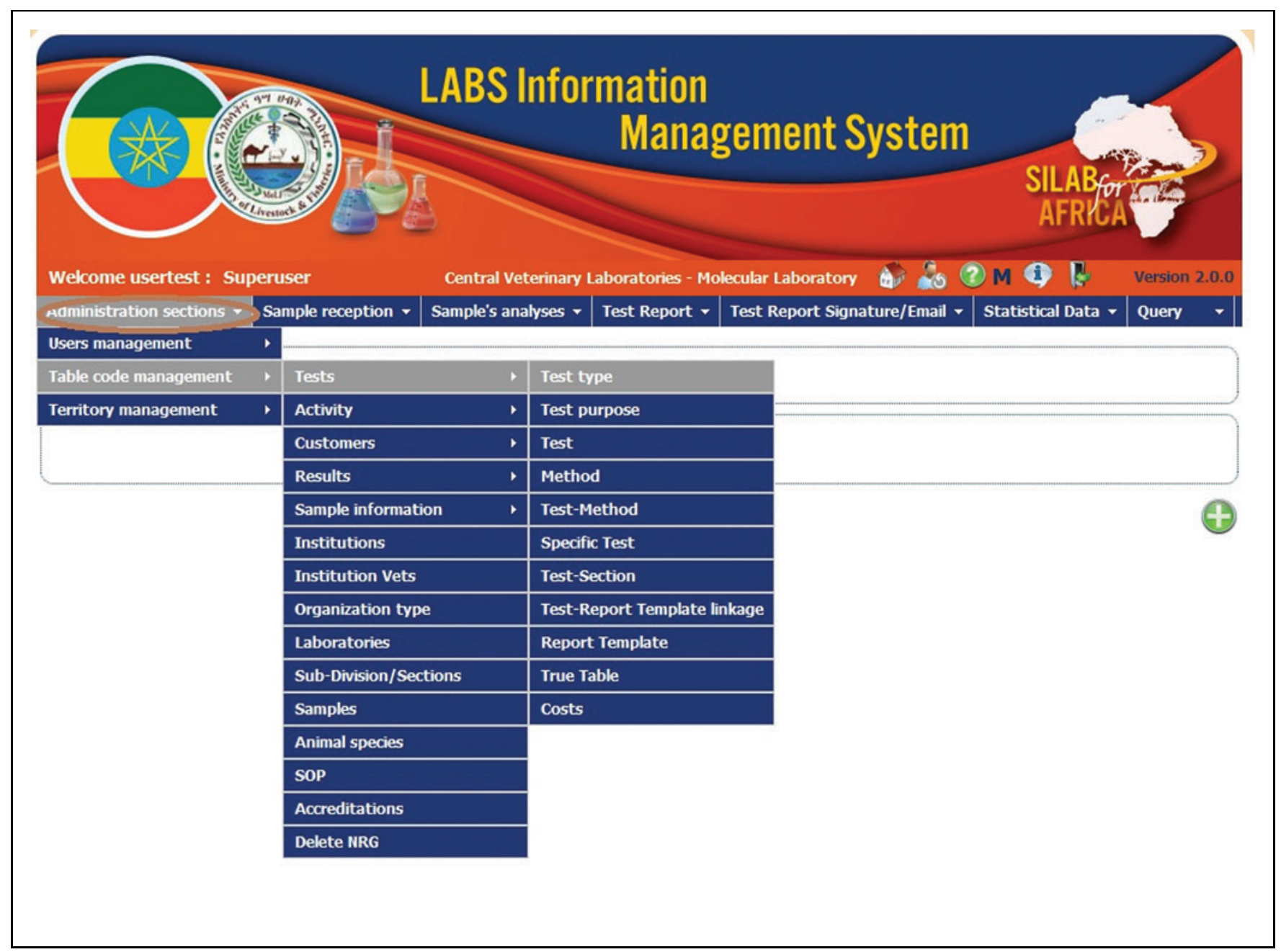

Fig. 6. Domain's tables.

According to that plan, all rabies cases have to be tested by official laboratories. SILABFA gathers additional epidemiological data regarding the sampling activity (Fig. 3), which relates to outbreak code, the number of animals affected, and their anamnesis and human cases involved. As described by the control plan, the alert system is based on e-mails sent to field veterinarians, with details on samples and test results. This system allows an immediate intervention to manage single cases; from the first analysis of reports, in 2016 the speed of intervention has increased to 50\%. Moreover, SILABFA acts like a collector of all useful data to monitor the national plan performance and analysis of results to evaluate its efficacy.

Another example of SILABFA data analysis was in Zimbabwe where brucellosis is endemic. Zimbabwe has a widespread dairy industry: dairy establishments were built in districts outside urban areas to improve milk availability in those remote areas. Using SILABFA data collected since 2012, the first attempt for estimating the prevalence of brucellosis in Zimbabwe was carried out. ${ }^{3}$

\section{Discussion and Conclusions}

Implementation of SILABFA in veterinary laboratories of African countries shows that each involved country made a remarkable improvement in laboratory management, considerable growth in service delivery, management and technical activities, improvement of quality of test reports, and an increase of data quality and consistency (Fig. 4).

Moreover, informative system's usage has simplified achievement and retention of laboratory's accreditation, by tracing all the phases of diagnostic process, from the arrival of samples to generation and signature of test reports (who doeswhat-when). 


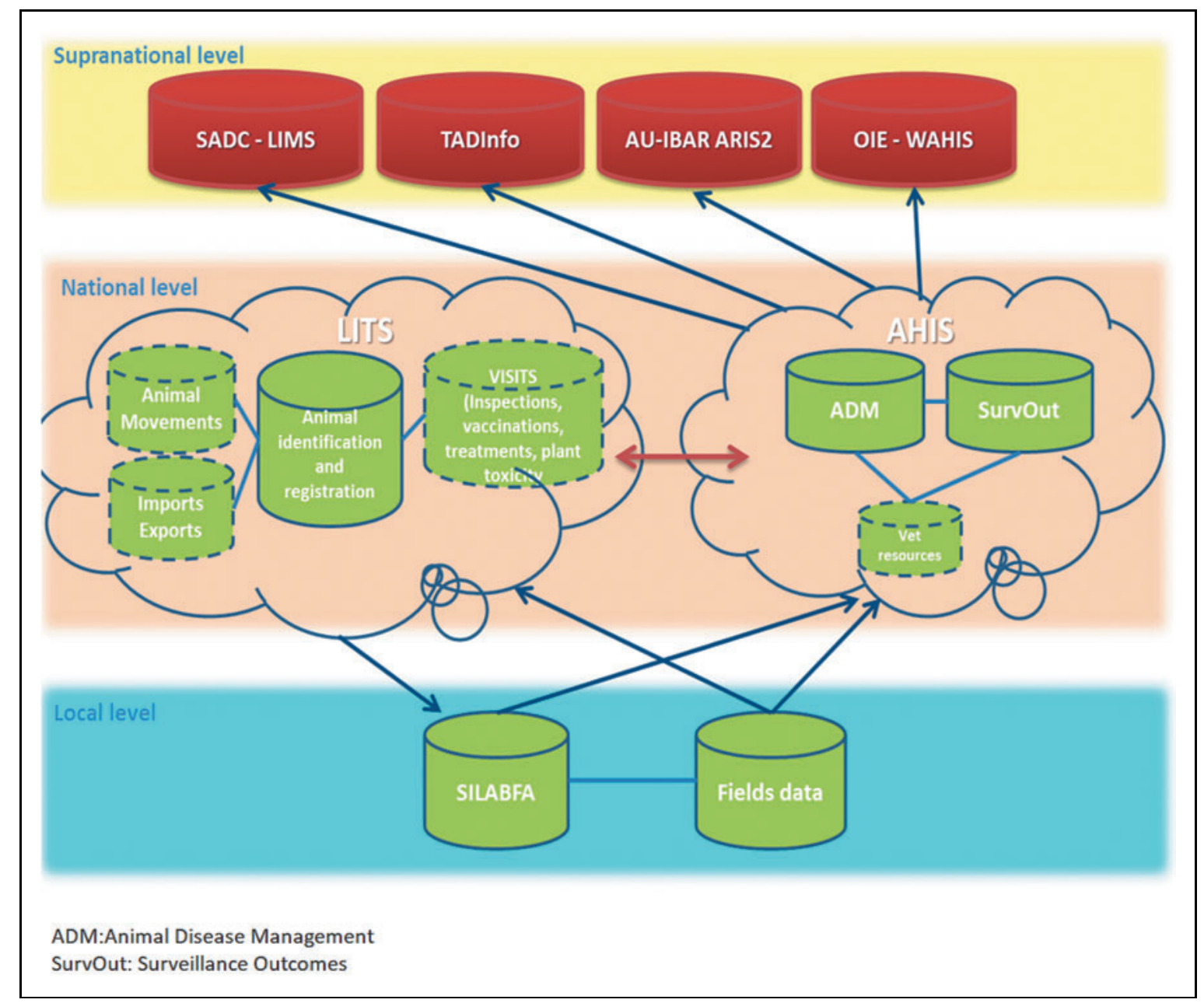

Fig. 7. AHIS data schema. AHIS, animal health information system.

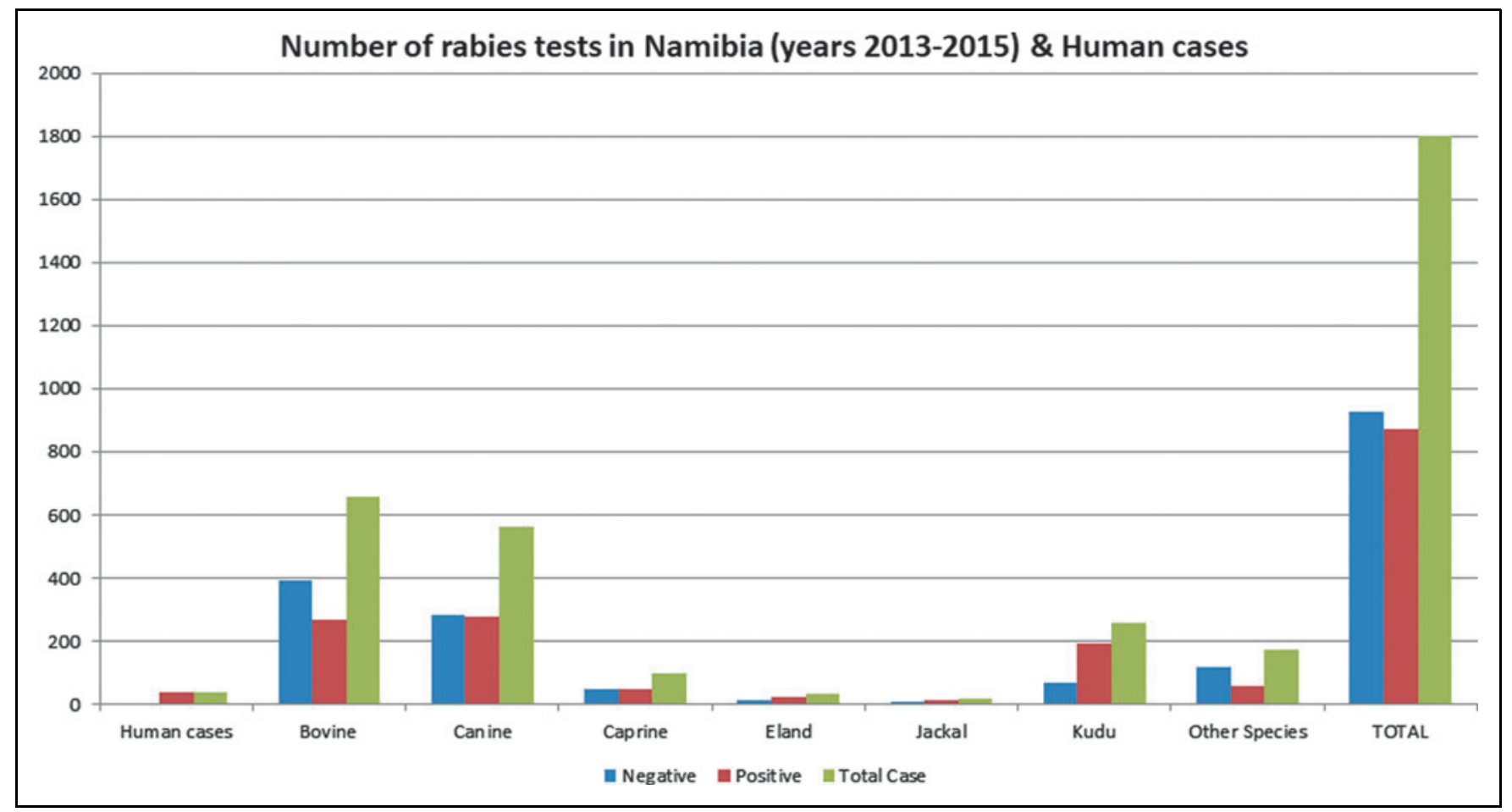

Fig. 8. Rabies in Namibia, animals and human cases. 


\section{COLANGELI ET AL.}

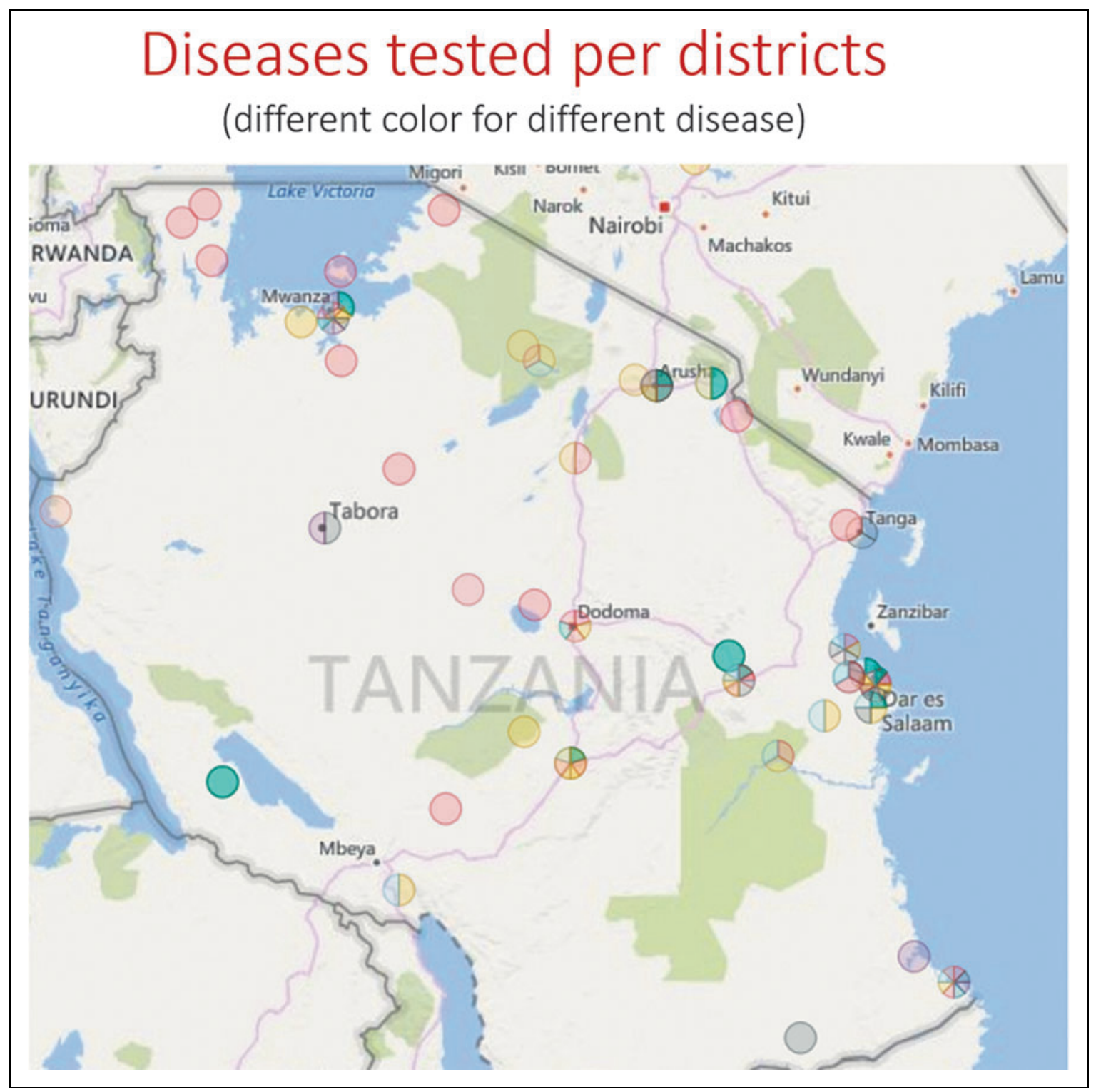

Fig. 9. Diseases tested per district.

An additional feature of SILABFA is to have statistics and to release automatic reports (Fig. 5). Periodical reports for African laboratories using SILABFA are fast and effective, and this feature has simplified the information flow among organizations.

SILABFA is very useful in the administration department because it produces automatic invoices and in the laboratory management because it monitors each step by avoiding delays in analysis and in test reports. The pushed usage of tables of codes (materials, species, tests, methods, and procedures) assures data quality and creates a unified semantics and language (Fig. 6).
However, some difficulties have been encountered: the most considerable regards the turnover of personnel already skilled specially in SILAB administrator role. For this reason, it is recommended to train at least two people in that role. In some countries, the availability of IT personnel at laboratory is not guaranteed, and staff have to wait for the expert from the ministry to solve simple problems such as restart the server.

Frequent power cutoffs, especially in peripheral laboratories and/or restricted internet connection time, limit SILABFA use and push people to prefer paper-based system. To overcome these obstacles, it is very important that the 
laboratory governance ensures the functioning requirements over time and the expert's team offers rapid technical support.

All difficulties described are "common" problems in African Laboratories (are the same regarding a new analytical equipment to be used). A specific critical point is regarding the quality of collected data. Initially, SILAB data are mainly used to produce test reports and internal alerts and statistics. In these cases, the data quality can be enough. Nevertheless, if you want to extract data for epidemiological studies, the accuracy of some information is to be improved (sampling data, animal vaccinations, symptoms, human cases, etc.) even if these data are not necessary for diagnosis.

SILABFA can not only be considered a simple laboratory management system ${ }^{4}$ but an effective and productive system of administration and an important database useful to epidemiologists to analyze health situations and study interventions in each country.

In countries where SILABFA and AIRTS are already present and integrated, it is possible to redesign a complete animal health information system (AHIS) to collect, manage, systematically analyze data, and to generate appropriate information for various stakeholders along livestock food value chains. These data may be used to support the decisionmaking process in relation to diseases prevention, eradication, or control, and may support the design, development, and management of surveillance programs in the framework of "One Health" approach.

Currently, the data of pertinence of an AHIS are collected by different not-integrated information systems and stored in various not interconnected databases. This fragmentation of systems and databases hampers an efficient data management with duplication of efforts and data entries and difficulties in producing epidemiological analyses and feedbacks for the stakeholders. An integrated AHIS (Fig. 7) must enable a correct integration of information produced by different information systems to avoid multiple entries of the same data. Significant efforts are required to standardize data and procedures to allow such coherent integration. The integrated AHIS supports the veterinary authorities in the decision-making process, providing worthy information on animal disease occurrence (Fig. 8), results of disease surveillance, and control activities (Fig. 9), and presence of significant risk factors influencing the diseases space time frequencies or trends. The AHIS system should be the next step in Namibia and Botswana where SILABFA is already linked to AIRT.

\section{Acknowledgments}

The authors thank the heads and all personnel of Namibia CVL, Botswana BNVL, Zambia CVRI, Zimbabwe CVL, Tanzania TVLA (HQ in Dar es Salaam, and Zonal Offices in Arusha, Iringa, Dodoma, Mwanza, Tabora, Mtwara, and Tanga), Uganda NADDEC, Kenya CVL and Kenya KALRO-VRI, Ethiopia NAHDIC, and Cameroon LANAVET. A particular thanks to Gwenaelle Dauphin, Beatrice Mouille, and Lidewij Wiersma FAO Animal Health Service $(A G A H)$ experts.

\section{Disclosure Statement}

No competing financial interests exist.

\section{REFERENCES}

1. Centers for Disease Control and Prevention (CDC). National center for emerging and zoonotic infectious diseases: Our work, our stories, 2011-2012. Atlanta, GA: CDC, 2013;55

2. Colangeli $P$, Ferrilli $M$, Quaranta $F$, Malizia $E$, Mbulu RS, Mukete $E$, lipumbu $L$, Kamhulu A, Tjipura-Zaire G, Di Francesco C, Lelli R, Scacchia M. Laboratory information management system: An example of international cooperation in Namibia. Vet Ital 2012;48:241-251.

3. Vhoko K, lannetti S, Vimbai Burumu J, De Massis F, Ippoliti C, Bhebhe B. Estimation of prevalence of Brucellosis in Cattle in Zimbabwe from samples submitted to the Central veterinary laboratory during the 2010-2014 period. Vet Ital 2018;54:21-27.

4. Colangeli $P$, De Massis F, Cito F, Mercante MT, Ricci L. Laboratory information management systems: Role in veterinary activities, In: Laboratory Management Information System: Current requirements and future perspectives. IGI-Global, 2014;297-309.

Address correspondence to:

Patrizia Colangeli, MS

Istituto Zooprofilattico Sperimentale dell'Abruzzo e del Molise "G. Caporale"-CIT

Via Campo Boario

Teramo 64100

Italy

E-mail: p.colangeli@izs.it

Received: July 29, 2018

Revised: November 15, 2018

Accepted: November 15, 2018

Online Publication Date: February 15, 2019 